\title{
A DWT-SWT based Image Super Resolution with Multi Surface Fitting
}

\author{
Gogireddy Sneha \\ M.Tech Student, \\ Department Of ECE \\ Vardhaman College of Engineering, \\ Telangana, India.
}

\author{
T.Ramakrishnaiah \\ Assistant professor (Sr.Grade) \\ Department of ECE \\ Vardhaman College of Engineering, \\ Telangana, India.
}

\begin{abstract}
In this paper, an image super-resolution technique is proposed which is based on interpolation of high frequency sub-band images obtained by Discrete Wavelet Transform on input image. In those sub-bands edges are enhanced by introducing an intermediate stage by using Stationary Wavelet Transform. The wavelet transform is applied in order to decompose image into different sub-bands. In those sub-bands, the high frequency sub-bands are interpolated and then these estimated high frequency sub-bands are modified by using high frequency sub-bands obtained through SWT. These all subbands are fused to generate a new high resolution by using inverse Wavelet transform techniques. The proposed results depict the conventional and state of art image resolution enhancement techniques.
\end{abstract}

Keywords: Image super resolution, Discrete and stationary wavelet transform.

\section{INTRODUCTION}

The term image super resolution when applied to sample images is related to information content has to be distinguished from size the size of an image. Super resolution is a technique that enhances the resolution of an image. In electronic imaging applications, image with high resolution are described. The high resolution means that pixel density within an image is high, and HR images can offer more details in various applications. This approach is to use signal processing techniques to obtain an HR image from the observed multiple low resolution images. A resolution enhancement approach has been one of the most active research areas and it is called super resolution image reconstruction or simply image resolution enhancement. An interpolation has been widely used in image super resolution reconstruction in many image processing applications such as super resolution [1]-[4]. For that image super resolution there are three well known interpolation techniques namely nearest neighbor interpolation, bilinear interpolation, bicubic interpolation. Nearest neighbor interpolation selects the value of nearest pixel by rounding coordinates of desired interpolation point. Bilinear interpolation algorithm interpolates from the nearest four mapped source pixels. Bicubic interpolation algorithms interpolate from the nearest sixteen mapped source pixels. Here we are using bicubic interpolation in our method.

Image super resolution enhancement in the wavelet domain is relatively new research topic and recently many existing algorithms have been proposed [4]-[6]. The wavelet transform technique used is one of the best wavelet transform techniques used in image processing. Discrete Wavelet Transform (DWT) decomposes image into various sub-band images namely low-low (LL), low-high (LH), high-low (HL), and high-high $(\mathrm{HH})$. This decomposing is done based on the concept of subband coding. Another recent wavelet transform which has been used in various image processing applications is stationary wavelet transform (SWT) [5]. The SWT is similar to DWT but it does not used down-sampling, the subbands will have size same as the input image.

\section{RELATED WORKS}

This paper proposes an image super resolution technique which generates sharper high resolution images. Proposed technique uses wavelet transform techniques to decompose a low resolution image into different sub-bands. The other three high frequency sub-band images have been interpolated using bicubic interpolation. Bicubic interpolation algorithms interpolate from the nearest sixteen mapped source pixels. The objective of this algorithm is to find the HR grid from a set of LR pixel frames after that those reference frames using the projective model for motion parameter estimation. After registering the LR pixels apply the sampling for irregular images. In order to create a digital image, we need to convert the continuous sensed data into digital form. To convert the image into digital form, we have to sample the function in both $\mathrm{x}$ and $\mathrm{y}$ coordinates and in Amplitude. Digitizing the coordinate values is sampling. The process can be given as

$$
\begin{gathered}
\Gamma=\Gamma\left(f\left(p_{L 1}\right), \ldots f\left(p_{L i}\right), \ldots . . f\left(p_{L K}\right), x_{L 1}, \ldots\right. \\
\ldots x_{L i}, \ldots . x_{L K}, y_{L 1}, \ldots \ldots y_{L i}, \ldots \ldots y_{L K} \\
f(p H)=S\left(x_{H}, y_{H}, \Gamma\right)
\end{gathered}
$$

Where $\Gamma$ is the surface fitted for multiple pixels. This algorithm converts this non-uniformly spaced raster to a uniformly spaced grid by multi-surface fitting by interpolation techniques. Initially the surface is (M X N) LR pixels are registered with respect to a chosen reference pixel. The basic principle for increasing the spatial resolution in SR techniques is the availability of multiple LR images is captured. In super resolution images represent various looks at the same scene on selected surface. The goal of this is to introduce the concept of SR algorithm for reconstructing the image with better quality. Before presenting the SR algorithms, we first model the LR image acquisition process. The SR image reconstruction is one of the most spotlighted research areas 


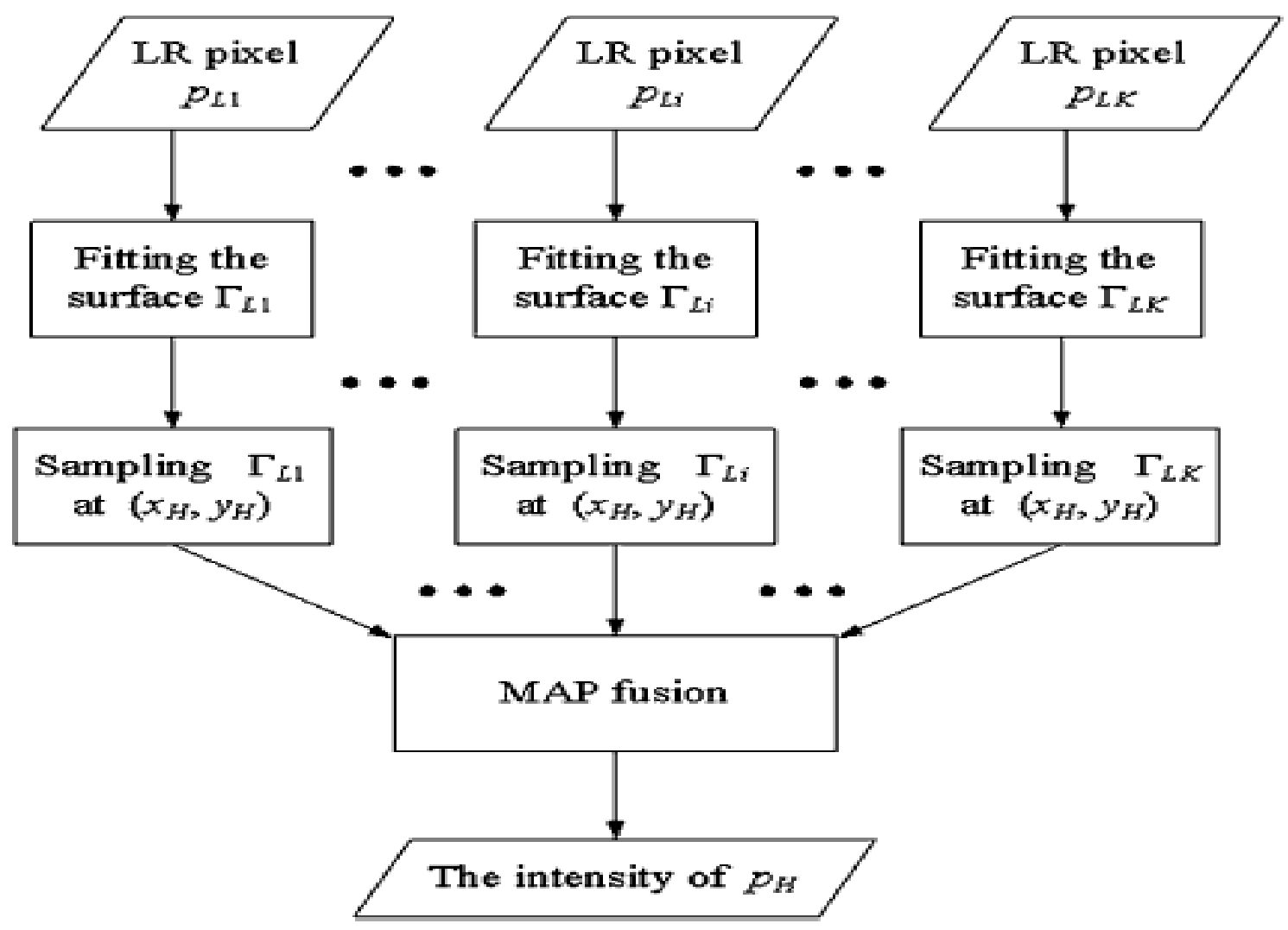

Fig 1 Flowchart to estimate High resolution pixel Intensity

and it can overcome the inherent resolution limitation of the imaging system and improve the performance of most digital image processing applications. In image resolution enhancement using interpolation the main loss is on its high frequency components occurred due to interpolation. To improve the quality of enhanced image preserving edges is essential. DWT is used to preserve these high frequency components of the image. The properties shift variance and redundancy of DWT mean that DWT coefficients are inherently interpolable.

\section{PROPOSED METHODOLOGY}

The proposed image resolution enhancement techniques by using interpolation have some limitations loss is on its high frequency components that is the edges are smoothing caused by interpolation. To increase the image quality of the super resolved images preserving the edges is required. This article proposes DWT which has been employed in order to preserve the high frequency components of the image. The DWT means that it decomposed coefficients are inherently interpolable. The corresponding one level DWT is used to decompose an input image into different sub-band images. In that four coefficients LL is approximation image and remaining three are horizontal(LH), vertical(HL) and Diagonal(HH). High frequency sub-bands (LH, HL, and HH) will contain the high frequency components of the input image. In this project we propose bicubic interpolation with enlargement factor if 2 is applied to high frequency sub-band images. On those sub-bands we are applying the down sampling. In each of the DWT sub-bands, ' information loss is occurred in the respective sub-bands. That's why SWT is employed to minimize these losses. The high frequency interpolated sub-bands and the SWT high frequency subbands have the same size which means that the same size can be added with each other. Then the corrected high frequency sub-bands can be interpolated for higher enlargement. It is known that the wavelet domain, the low resolution image is obtained by low pass filtering of high resolution image. Instead of using the low frequency sub-bands which contains less information than the original high resolution images; here we are using the input image for the interpolation of the low frequency sub-band images. The input image by N/2 and high frequency sub-bands by 2 and $\mathrm{N}$ in the intermediate and final interpolation stages respectively and then applies IDWT as illustrated in the fig2. If the output image getting sharper edges then directly apply the interpolation of the input image. Due to the fact of interpolation is isolated high frequency components are in high frequency sub-bands corrected by adding the high frequency sub-bands of SWT of the input image will preserve more high frequency components after the interpolation than interpolating input image directly.

\section{RESULT AND ANALYSIS}

The input images have low resolution obtained by down sampling the original high resolution images. The effectiveness of the proposed method over the conventional and state of art image resolution enhancement techniques for best well known test images (Lena, Cameraman) with different features are used for comparison. Here we will compare the PSNR performance of the proposed technique using the bicubic interpolation with conventional and state-of art resolution enhancement techniques. The proposed technique over performs the conventional and state of art image super resolution enhancement techniques. 


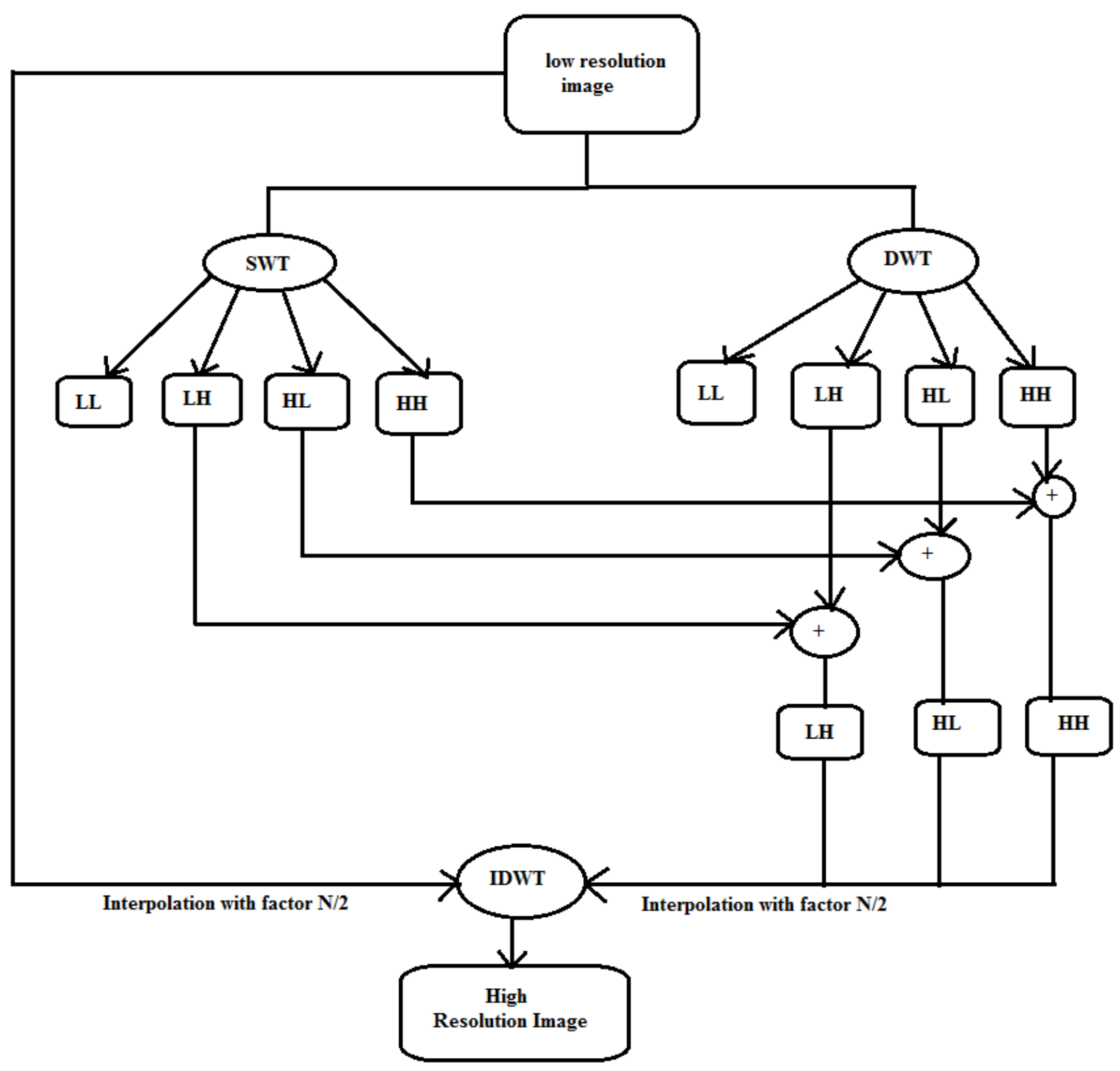

Figure 2. Block Diagram of the Proposed Super Resolution Algorithm.

\section{EXPERIMENTAL RESULTS}

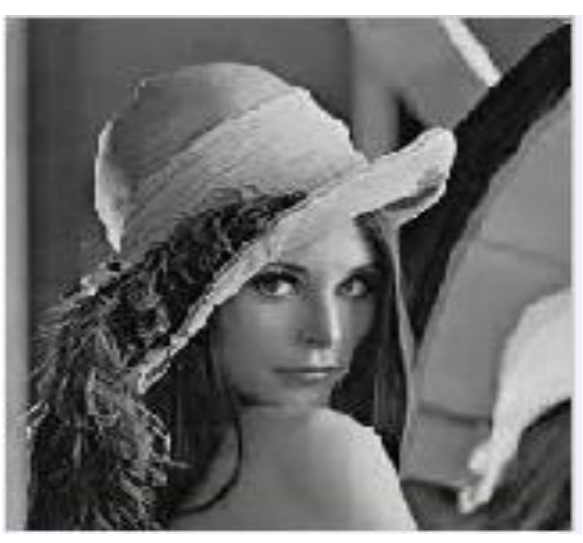

Figure 3 Input image

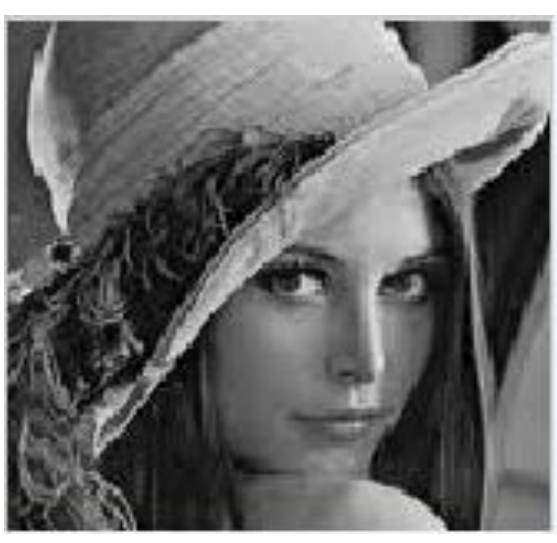

Figure 4 Cropped image 


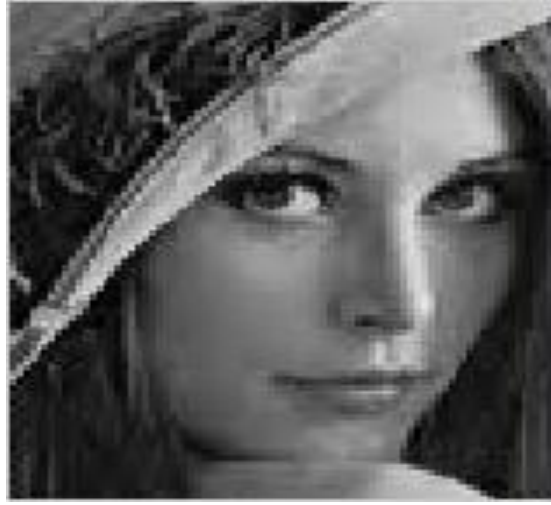

Figure 5 Cropped of cropped Image

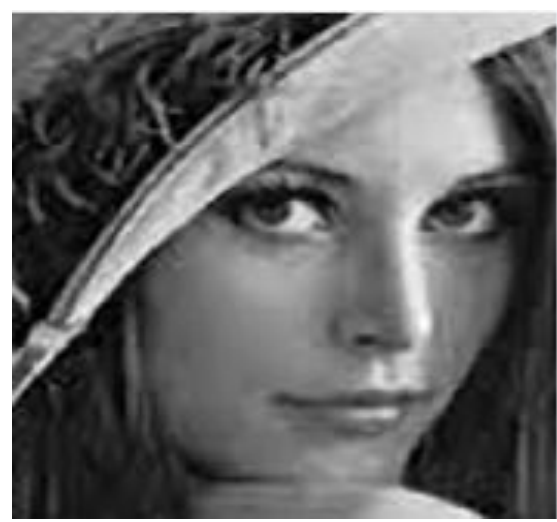

Figure 7 Interpolated Image

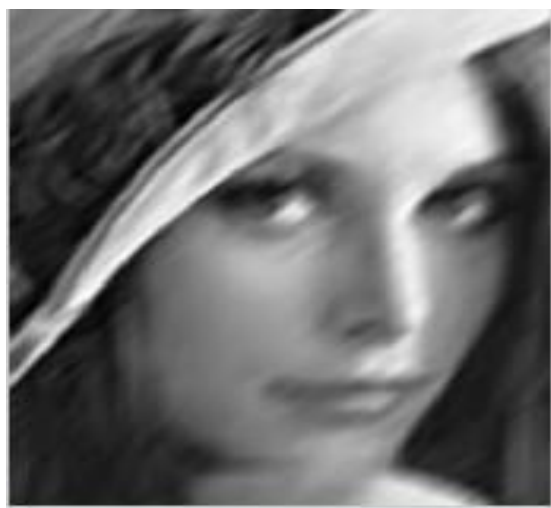

Figure 6 Expanded Image

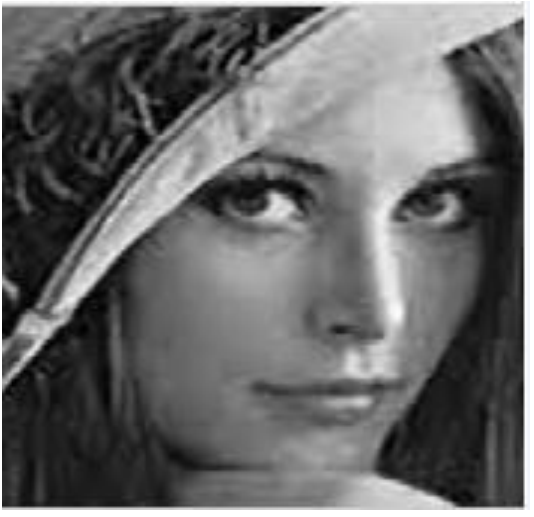

Figure 8 Output Image

Table 1: PSNR and MSE Results of Proposed Technique

\begin{tabular}{|c|c|c|}
\hline Parameter & Interpolated Image & DWT-SWT Image \\
\hline Mean Square Error & 57.19 & 0.042 \\
\hline Peak Signal Noise Ratio & 30.59 & 61.88 \\
\hline
\end{tabular}

\section{CONCLUSION}

In this paper, we have presented a image SR reconstruction framework using multisurface fitting. It creates one surface for every LR pixel. These surfaces can effectively retain the image details such as image gradients, curvatures, or even higher order information. Each surface has different weights in estimation of the HR intensity values. In the MAP frame, the surfaces with smaller noise and errors tend to have greater contributions. An original image is interpolated with half of the interpolation factor used for interpolation the high frequency sub-bands. Then all these images have been combined using IDWT to generate a super resolved image. The proposed technique has been tested on well known images, where their visual results show the superiority over conventional image enhancement techniques.

\section{REFERENCES}

[1] H. Demirel, G. Anbarjafari, and S. Izadpanahi, "Improved motionbased localized super resolution technique using discrete wavelet transform for low resolution video enhancement," in Proc. $17^{\text {th }}$ Eur. Signal Process. Conf., Glasgow, Scotland, Aug. 2009, pp. $1097-$ 1101.

[2] Y. Piao, I. Shin, and H. W. Park, "Image resolution enhancement using inter-subband correlation in wavelet domain," in Proc. Int. Conf. Image Process., 2007, vol. 1, pp. I-445-448.

[3] H. Demirel and G. Anbarjafari, "Satellite image resolution enhancement using complex wavelet transform," IEEE Geoscience and Remote Sensing Letter, vol. 7, no. 1, pp. 123-126, Jan. 2010.

[4] C. B. Atkins, C. A. Bouman, and J. P. Allebach, "Optimal image scaling using pixel classification," in Proc. Int. Conf. Image Process., Oct. 7-10, 2001, vol. 3, pp. 864-867. 
[5] J. E. Fowler, "The redundant discrete wavelet transform and additive noise,"Mississippi State ERC, Mississippi State University, Tech. Rep. MSSU-COE-ERC-04-04, Mar. 2004.

[6] S. Mann and R. W. Picard, "Video orbits of the projective group: A simple approach to featureless Processing, vol. 6, no. 9, pp. 1281-1295, September 1997. [7] S. Lertrattanapanich and N. K. Bose, "Latest Results on High-Resolution Reconstruction From Video Sequences, Technical Report of IEICE," The Institution of Electronic, Information and Communication Engineers, Japan, DSP99-140, 1999.

[8] N. K. Bose and K. J. Boo, "High-resolution image reconstruction with multisensors," International Journal Imaging Systems and Technology, vol. 9, pp. 294-304, 1998 .
[9] M. K. Ng, J. Koo, and N. K. Bose, "Constrained total least-squares computations for high-resolution image reconstruction with multisensors," International Journal Imaging Systems and Technology, vol. 12, pp. 35-42, 2002.

[10] N. Nguyen and P. Milanfar, "A wavelet-based interpolation-restoration method for superresolution (wavelet superresolution)," Circuits Systems Signal Process, vol. 19, no. 4, pp. 321-338, 2000.

[11] N. K. Bose and S. Lertrattanapanich, "Advances in wavelet superresolution," in SAMPTA 2001: Proceedings of International Conference on Sampling Theory and Application, May 13-17, 2001, pp. 5-12.

[12] L. Poletto and P. Nicolosi, "Enhancing the spatial resolution of a two-dimensional discrete array detector," Opt. Eng., vol. 38, no. 10, pp. 1748-1757, October 1999. 